\title{
SKYRMIONS AND THE NUCLEAR FORCE
}

\author{
Niels R. Walet and R. D. Amado \\ Department of Physics, University of Pennsylvania, Philadelphia PA 19104-6396
}

\begin{abstract}
The derivation of the nucleon-nucleon force from the Skyrme model is reexamined. Starting from previous results for the potential energy of quasistatic solutions, we show that a calculation using the Born-Oppenheimer approximation properly taking into account the mixing of nucleon resonances, leads to substantial central attraction. We obtain a potential that is in qualitative agreement with phenomenological potentials. We also study the non-adiabatic corrections, such as the velocity dependent transition potentials, and discuss their importance.
\end{abstract}

21.30.+y, 13.75.Cs, 12.40.-y, 11.10.Lm

Typeset Using REVTEX 


\section{INTRODUCTION}

A central problem of strong interaction theory is to derive the nucleon-nucleon interaction from more fundamental principles. It is well established that quantum chromodynamics (QCD) is the correct theory of strong interactions. The phenomenology of the nucleonnucleon interaction suggests a description in terms of nucleons and the exchange of mesons, while QCD is a non-abelian gauge theory of quarks of three colors interacting by exchange of gluons. These two pictures, one of quarks and gluons and the other of nucleons exchanging mesons are difficult to reconcile. The notions of asymptotic freedom and its counterpart confinement suggest that quarks and gluons are the appropriate degrees of freedom for short distances and high energy, but are not for the large distances and relatively low energies of the nucleon interaction, where the quark picture becomes strongly non-perturbative. The problem is to make a theory, starting from QCD, that obtains the nucleons and their meson exchange. If such a theory agrees with the vast body of nucleon-nucleon interaction phenomenology, it will have, in some sense, "derived" traditional nuclear physics from QCD. This paper reports the details of an effort based on the Skyrme model [1,2] to make that connection. A brief summary of these results have been presented elsewhere [3].

The Skyrme model describes a nonlinear, classical field theory of interacting pions. This seems far from QCD, but in fact the Skyrme Lagrangian has been shown to be an effective theory of QCD in the large distance (long wave length) non-perturbative regime in the limit of a large number of colors $\left(N_{\mathrm{C}}\right)$. There is no unique path from such a classical theory back to quantum chromodynamics with three colors, but attempts to construct alternate classical pictures have shown that there is surprisingly little leeway [4], and work to extract nucleon phenomenology from the Skyrme Lagrangian has been relatively successful with results that are fairly insensitive to variations in details of the theory. Hence the Skyrme Lagrangian provides a robust, QCD-based starting point for studies of nucleon and nucleon-nucleon properties in the long wavelength, non-perturbative regime.

The Skyrme Lagrangian possesses a topologically conserved charge, $B$, that Skyrme [1] identified with baryon number. The $B=1$ solutions of the Skyrme Lagrangian yield an appealing picture of the nucleon and its excited states, but since the theory is a large $N_{\mathrm{C}}$ approximation, corrections for real nucleon observables are expected to be of order $1 / N_{\mathrm{C}}$ or $\sim 30 \%$. An interesting and important problem area is the calculation of these $1 / N_{\mathrm{C}}$ corrections. These are first quantum corrections, and their calculation faces the standard uncertainties found in going from any classical theory, particularly a non-linear field theory, to a quantum theory.

The relative success of the Skyrme picture for the $B=1$ system invites its extension to $B=2$ and in particular to the problem of the nucleon-nucleon interaction. The phenomenology of the $N N$ interaction suggests that, with the possible exception of the repulsive core, the interaction is well described in terms of multiple pion exchange. Thus the Skyrme model which expresses the physics in terms of interacting pions, seems to be a good starting point. Skyrme himself [1] noted that for very large separation, the $N N$ interaction reduces to one pion exchange in his model. The key question then is whether the model can also give the central mid-range attraction that is responsible for nuclear binding. There are three problems that must be overcome in addressing this question. The first is the solution of the classical $B=2$ field equations to obtain and describe $B=2$ configurations. The 
second is the problem of obtaining nucleon-nucleon interactions from Skyrmion-Skyrmion interactions. The third is the introduction of $1 / N_{\mathrm{C}}$ corrections. As we shall see the last two are inexorably mixed. The need for careful $1 / N_{\mathrm{C}}$ corrections is much greater in the $B=2$ problem than for $B=1$, because the only way presently known to calculate the interaction energy is to calculate the energy of the total $B=2$ system and then subtract the energy of two free $B=1$ Skyrmions. This is a difficult, delicate, and dangerous process since the interaction energy is a very small fraction of the total. In this paper we present a procedure for carrying out the three steps. Our procedure yields a $N N$ interaction closely resembling standard phenomenology. The interaction has a one pion exchange tail at long distance, a short range repulsion, a tensor force and, best of all, a strong, mid-range central attraction-all in substantive agreement with phenomenology.

The problem of solving or even of describing the $B=2$ interacting Skyrmion system is complex. There are only two known stable (time-independent) $B=2$ configurations. One is the trivial one of two infinitely separated $B=1$ Skyrmions, and the other is a compact bound state of toroidal baryon density in which the identity of the two individual Skyrmions is lost. To obtain an interaction energy one must define a set of intermediate, unstable, static Skyrmion configurations at finite separation. Each configuration defines a point on an adiabatic, six dimensional, collective manifold, called the unstable manifold by Manton [5]. Each point on the manifold is stabilized by imposing a constraint. The dimensionality of this manifold makes it virtually impossible to calculate the interaction energy at every point. Fortunately much of the interaction energy can be described by studying only a very small part of that manifold. But on that sub-manifold the description must be accurate. As we emphasized above, the interaction energy is calculated as the difference between large numbers. Hence seemingly small inaccuracies in configurations can lead to large discrepancies in interaction energy. It is this problem that has plagued early attempts to study the $N N$ interaction in the Skyrme model with the product ansatz. This ansatz, also first given by Skyrme [1], describes the $B=2$ system as the product of two $B=1$ systems. The configurations so obtained are not solutions of the constrained nonlinear field equations, and hence the energy they yield is above the true energy. For very large separation these differences are not serious, but for intermediate and short distances they are fatal. For example the product ansatz cannot describe the toroidal lowest $B=2$ state. Early efforts [6.7] to study the $N N$ system with the product ansatz developed much of the vocabulary and sense of the problem, but in the end failed to give any mid-range attraction. Surprisingly enough one finds a repulsive core in this ansatz, but it has been shown that this does not have the correct g-parity [8]. A far better approach to the $B=2$ system is direct numerical solution of the constrained, non-linear, field equations. Recently Walhout and Wambach (WW) [9] have produced just such solutions. They form the starting point of our work.

How does one go from the Skyrmion-Skyrmion interaction energy calculated on the interesting sub-manifold of the unstable manifold to the $N N$ interaction? A commonly used prescription is to project the Skyrmions to nucleons at each separation. This method has been used extensively in the early product ansatz work. Using the same approach WW find weak central attraction. The nucleons only requirement is too strong, however. Phenomenologically all that is required is that the particles be nucleons asymptotically. When they approach and interact they can deform and be excited, for instance to the $\Delta$ 
state, as the dynamics dictates. This state mixing must be very important, since in the solutions for the interacting Skyrmions found by WW, the individual Skyrmions deform strongly as they interact, finally loosing their individuality completely in the toroidal state. If the time scales (or equivalently energy scales) for the radial motion and the excitation of Deltas separate, the contribution of this distortion to the energy can be calculated in the Born-Oppenheimer approximation. Finite $N_{\mathrm{C}}$ effects are critical in making the time scales appropriately distinct. It is this combination of finite $N_{\mathrm{C}}$ effects and Born-Oppenheimer that is crucial to obtaining the phenomenological potential. Each idea has been used before [10], but it is the combination of exact solution of the classical field equations, state mixing using the Born-Oppenheimer approximation and finite $N_{\mathrm{C}}$ effects that together makes physical sense and gives the phenomenologically correct potential as we shall see below. We shall first treat the effects of the distortions perturbatively, where it is clear that the distortion makes an attractive contribution. Since the contribution of the distortions becomes large at small distances, we turn to diagonalization of the Born-Oppenheimer Hamiltonian to check the perturbative results. Once we make the Born-Oppenheimer separation we can calculate explicit corrections to the adiabatic picture. These take the from of transition potentials between the different adiabatic channels, as well as some velocity dependent potentials similar to Berry's phase terms.

In Section II we set out some key notions. We review the Skyrme Lagrangian, the adiabatic manifold for interacting $B=2$ Skyrmion systems, review the algebraic method for projecting baryon states from Skyrmions and for introducing finite $N_{\mathrm{C}}$ effects, and show how these algebraic methods permit a description of the Skyrmion-Skyrmion interaction on the unstable manifold. This section brings together the known tools we need for the problem. Section III presents our derivation of the $N N$ interaction from the Skyrmion interaction for finite $N_{\mathrm{C}}$. It gives the adiabatic interaction derived first in perturbation theory and then using the Born-Oppenheimer approximation. The formalism for obtaining the transition potentials is also given here. Our results are presented in Section IV. As we have discussed above, the potentials are close to static $N N$ potentials obtained from the data. Section V gives a brief summary and conclusions. Some of the algebraic details of the perturbation treatment are presented in the appendix.

\section{SOME KEY NOTIONS}

\section{A. The Skyrme model}

The Skyrme model is a non-linear field theory that can be realized in terms of an $\mathrm{SU}(2)$ valued matrix field $U$, with Lagrangian density

$$
\mathcal{L}=\frac{f_{\pi}^{2}}{4} \operatorname{Tr}\left[\partial_{\mu} U(x) \partial^{\mu} U^{\dagger}(x)\right]+\frac{1}{32 g^{2}} \operatorname{Tr}\left[U^{\dagger} \partial_{\mu} U, U^{\dagger} \partial_{\nu} U\right]^{2} .
$$

The model is covariant, as well as invariant under global SU(2)-rotations that are identified with the isospin symmetry. As was discovered by Skyrme the model has a topologically conserved quantum number, which is identified as the baryon number $B$. The $U$ field is interpreted as a combination of a scalar $\sigma$ field and an isovector pion field, $U=\sigma+i \vec{\tau} \cdot \vec{\pi}$. The $\sigma$ field is not an independt physical field due to the unitarity constraint on $U$. 
The standard time-independent solution to the classical field equations for $B=1$ is the defensive hedgehog, where the pion field points radially outward,

$$
U_{1}(\vec{r})=\exp (i \vec{\tau} \cdot \hat{r} f(r)) .
$$

The baryon number of this state is given by $B=(f(0)-f(\infty)) / \pi=1$. This solution breaks translational invariance, as well as the $O(4)$ spin-isospin symmetry. If we perform a global $\mathrm{SU}(2)$ isorotation on the state,

$$
U_{1}(\vec{r} \mid A)=A^{\dagger} U_{1}(\vec{r}) A
$$

we obtain a state of the same energy. One can easily show that an isorotation $A$ has the same effect on $U$ as performing an ordinary rotation $R\left[A^{-1}\right]$ on $\hat{r}$, so that the grandspin $K$, the sum of spin and isospin, is a good quantum number, with eigenvalue 0 ,

$$
\vec{K}=\vec{S}+\vec{I}
$$

In the $B=2$ system we shall frequently use the product ansatz. This ansatz makes use of the fact that the product of two $B=1$ solutions has baryon number two. The most general ansatz we can therefore construct from two hedgehogs consists of the product of two rotated hedgehogs, where the centers have been translated,

$$
\begin{aligned}
U_{2}(r \mid \vec{R} A B) & =A^{\dagger} U_{1}(\vec{r}-\vec{R} / 2) A B^{\dagger} U_{1}(\vec{r}+\vec{R} / 2) B \\
& =U_{2}(r \mid \vec{R} C D) \\
& =D^{\dagger} C^{1 / 2 \dagger} U_{1}(\vec{r}-\vec{R} / 2) C U_{1}(\vec{r}+\vec{R} / 2) C^{1 / 2 \dagger} D .
\end{aligned}
$$

In the last of (5) we have introduced the matrix $D$, that describes the rigid isorotation of the whole system, as well as a relative isorotation $C$. When $R$ is very large changing $C$ or $D$ does not change the energy of the solution. For smaller $R, D$ still generates a zero-mode (corresponding to broken isospin symmetry), but the energy will depend on $C$. Again, the energy is also invariant under spatial rotation, due to the conservation of angular momentum $\vec{J}=\vec{L}+\vec{S}$.

\section{B. Determination of manifold}

After having constructed all the constrained $B=2$ solutions, we construct a manifold that contains all these solutions. This method, which is well know in nuclear structure physics as the adiabatic large amplitude collective motion [11], seems first to have been introduced in the theory of interacting Skyrmions by Manton [5], under the name unstable manifold. His discussion is based on the deep geometric results by Atiyah and Hitchin for the interaction of BPS monopoles [12], which make an identification of the structure of the manifold very clear but do not give many tools for constructing it.

One of the ways to solve for the adiabatic collective manifold would be to solve a local fluctuation equation. This is a very complex problem for a field theory, but even when we reduce the complication by selecting only a finite number of degrees of freedom as in the Atiyah-Manton ansatz 13, 14 this remains a formidable (but interesting) task. The 
minimal set of degrees of freedom can be identified without performing any calculation, however. There are six trivial ones, consisting of the three center of mass coordinates, as well as global rotations of the whole system (in both space and isospace), which we shall ignore in the present discussion. For large separations we find that an additional six degrees of freedom are necessary to describe the product ansatz (5): the relative separation $\vec{R}$ (three coordinates) and three more coordinates describing the relative orientation (as discussed in the previous subsection usually represented by the $U(2)$ matrix $C=c_{4} I+i \vec{c} \cdot \vec{\tau}$, where $c_{4}^{2}+\vec{c}^{2}=1$ ). The minimal degrees of freedom are just the continuously deformed set of six parameters as we take $R$ to smaller values. The meaning of the word "adiabatic" in adiabatic manifold is that the motion in the manifold consists of the slowest modes. This only makes sense if at any point all the unstable directions lie within the collective space, so that no lower energy mode lies outside the manifold. An analysis of the unstable $B=2$ hedgehog by Manton [5] shows that it is plausible that this state has six unstable modes, again corresponding to relative separation (three modes) and a relative rotation (another three). Since this hedgehog is supposed to be part of the collective surface, we again find a minimum of six collective modes.

Once one determines the collective surface there exist standard techniques [11] to determine the collective Hamiltonian, with the restriction that it is quadratic in momenta. Schematically it takes the form

$$
H=K\left(R, \hat{R}, C, p_{R}, L_{i}, p_{C j}\right)+\frac{1}{2} I_{i} M_{i j} I_{j}+\frac{1}{2} J_{i} N_{i j} J_{j}+V(R, \hat{R}, C)
$$

Here $p_{R}$ is the momentum associated with $R, L$ the orbital angular momentum and $p_{C}$ is shorthand for the momentum associated with $C$. The symbols $I_{i}$ and $J_{i}$ denote the intrinsic components of the (conserved) isospin and angular momentum, respectively. The kinetic energy is closely related to the geometric structure of the collective surface, and can be very complicated. One example that has been studied extensively is the case of the BPS monopole, where one has only kinetic and no potential energy. It has been proven [12] that the underlying geometry is reasonably complicated and allows for non-trivial scattering of monopoles. In the current work we concentrate on the potential energy, however. We assume that the kinetic terms (including the contributions of $I$ and $J$ ) take the form

$$
K=\frac{1}{2 M} \vec{p}_{R}^{2}+\frac{1}{2 \mathcal{I}}\left(C_{2}^{1}+C_{2}^{2}\right),
$$

where $C_{2}^{i}$ denotes the $O(4)$ Casimir operator $\frac{1}{2}\left(S_{i}^{2}+I_{i}^{2}\right)$. The only correction to this that has been studied is the $R$ and $C$ dependence of $M$ [20], but we ignore that in this paper. It will be interesting to return to this problem in the context of the $N N$ interaction.

To calculate the manifold beyond the product ansatz we use the fact that for special choices of $C$, due to symmetry, there exist three stable situations [14 - the stable attractive channel, the marginally stable hedgehog-hedgehog and repulsive channels - where a path of the collective surface is traced out for fixed $C$. In each of these channels (labeled by $i$ for convenience) one calculates the classical potential energy $V_{i}$. The identification of the symbols $R$ in each of the three Hamiltonians as one and the same radial coordinate is a bit precarious. There is no apparent reason why we cannot separately redefine the radial coordinate in each channel (say, by a point transformation). Here the topology comes 
into play however. Since each channel corresponds to a definite choice of $C$, the three point transformations can be embedded in a single transformation that depends on both $R$ and $C$, so that we are just making a change of coordinate transformation on the manifold, by which we do not gain much. In order to sensibly identify the $R$ 's in the same channel we should use geometric notions. First note that each of the three channels $i$ seems to correspond to a geodesic line on the collective surface, due to the extra symmetry of the pion field. In order to be able to make a meaningful identification of points on the three lines as having the same $R$ the three points should lie on a single geodesic surface on the manifold, that crosses the three geodesic lines $i$ orthogonally. This is of course extremely difficult to implement, and we do not know of a good way of doing this. In most discussions of the Skyrmion-Skyrmion interaction, including the present one, these problems are ignored, and a special definition of $R$ is made that is then used to identify the different channels.

\section{The algebraic model}

Having determined the functions $V_{i}$ we need to understand their relation to the nucleonnucleon potential. The usual approach is to return to the product ansatz, where one makes very strong assumptions about the form of the two-Skyrmion solution. Again, this form is exact in the large- $R$ limit. The advantage of this form is that it guides us into a particular expansion of $V$ in terms of functions $\vec{c} \cdot \hat{R}, c_{4}$ (the quaternion $c$ specifies the relative orientation). This expansion is closely related to an algebraic model of the Skyrmion [15], which in the large- $N$ limit behaves in exactly this manner. There is no a priori reason why this form should be restricted to the product ansatz, however. As we have argued above the coordinates of the collective surface are in one-to-one correspondence with those of the product ansatz, so that the current problem can again be modeled by a similar form [17.

Let us now discuss the algebraic model [18, 15 that we use. Originally it was introduced to give an algebraic alternative to the rotational zero-mode quantization of the Skyrmion. Since the Skyrmion breaks $S O(4) \sim S U_{I}(2) \times S U_{S}(2)$ symmetry, but is invariant under a $S O(3)$ subgroup, we find that we can describe each of the equivalent configurations by a point of on the sphere $S_{3} \sim S O(4) / S O(3)$ [19].

We can now introduce a bosonic model with dynamical symmetry $S U(4)$, which in the limit of large boson number is also described by a point on the sphere $S_{3}$. Without discussing the hamiltonian in any detail, we can draw some interesting conclusions. For any $N$ (the number of bosons) we find that, as in the Skyrme model, all states have $I=S$. The spectrum of the algebraic model is cut off at $I=N / 2$, however. Since we believe that in the real world, where $N_{\mathrm{C}}=3$, we have a cut-off at isospin $3 / 2$ for the delta, we impose the condition that the number of bosons $N$ is equal to the number of colors $N_{\mathrm{C}}$. If we make this identification one can immediately show that for $N=N_{\mathrm{C}}=3$ the algebraic model is identical to the non-relativistic quark model without radial excitations. It has been shown that this model can be used to extract finite $N_{\mathrm{C}}$ corrections to observables. Of course we quantize only a few coordinates and we can only calculate a very limited set of finite $N_{\mathrm{C}}$ corrections, corresponding to the zero-mode quantization. One may hope, however, that through a judicious choice of collective coordinates we have found the most important, and maybe even the largest, contributions.

In this paper we are not interested in the single Skyrmion case, but rather in the the case 
of two Skyrmions. This has been discussed in quite some detail for the case of the product ansatz in Ref. [15]. The algebraic model used consists of two sets of $u(4)$ algebras, one for each Skyrmion, as well as a radial coordinate $\vec{R}$. The interaction of the two Skyrmions can be expanded in terms of three operators, the identity $I$, and the operators $W$ and $Z$ :

$$
\begin{aligned}
W & =T_{p i}^{\alpha} T_{p i}^{\beta} / N_{\mathrm{C}}^{2}, \\
Z & =T_{p i}^{\alpha} T_{p j}^{\beta}\left[3 \hat{R}_{i} \hat{R}_{j}-\delta_{i j}\right] / N_{\mathrm{C}}^{2} .
\end{aligned}
$$

Here $\alpha$ and $\beta$ label two different sets of bosons, used to realize the $u(4)$ algebras, and $T$ is a one-body operator with spin and isospin 1 . The semi-classical (large- $N_{\mathrm{C}}$ ) limit of these operators can be given in terms of $\hat{R}$ and $C=c_{4} I+\vec{c} \cdot \vec{\tau}$ as [15]:

$$
\begin{aligned}
W_{\mathrm{cl}} & =3 c_{4}^{2}-\vec{c}^{2}, \\
Z_{\mathrm{cl}} & =6(\vec{c} \cdot \hat{R})^{2}-2 \vec{c}^{2} .
\end{aligned}
$$

If we expand the Skyrmion-Skyrmion interaction we find

$$
v(R)=v_{1}(R)+v_{2}(R) W_{\mathrm{cl}}+v_{3}(R) Z_{\mathrm{cl}}+\text { higher order. }
$$

Fortunately studies of the $B=2$ system with the product ansatz [6,07, 15], the Atiyah-Manton ansatz [13, 14] and with direct numerical integration of the equations [9] have shown that much information about the $B=2$ energy as a function of separation $R$ is contained in the particular choice of three paths discussed earlier, corresponding to three definite choices of the relative orientation, or equivalently to a special symmetry of the pion field. This allows us to calculate an approximate form for the interaction energy of two Skyrmions which consist of the first three term of (10) (the form used in Ref. [9] is fully equivalent), which correspond to an expansion up to first order only in terms of the two scalar operators $W$ and $Z$. One of the important consequences of the explicit realization of these operators is that now algebraic methods [15,18] can be used to obtain their explicit finite $N_{\mathrm{C}}$ realization. In this way we can include some (arguably the most important) finite $N_{\mathrm{C}}$ corrections in the calculation. This includes the well-known amplification of the nucleon-nucleon matrix element of $T$ by the factor $\left(N_{\mathrm{C}}+2\right) / N_{\mathrm{C}}$.

\section{THE ADIABATIC INTERACTION}

The problem we address here is how to obtain a nucleon-nucleon interaction which includes some finite $N_{\mathrm{C}}$ effects, from (10). To date most workers have simply sandwiched (10) between states containing two nucleons for each value of $R$ and called that the interaction. This is definitely not the nucleon-nucleon interaction determined phenomenologically from scattering phaseshifts, where we can only require that the state contains two nucleons at large separation. For shorter distances, when the hadrons are interacting they can be (virtually) what ever the dynamics requires, for example $\Delta$ 's. We know [14] that the baryon density is strongly deformed for moderate $R$ - a deformation that finally results in the toroidal shape with its $70 \mathrm{MeV}$ of binding. That configuration corresponds to a great

deal of mixing between all states of the individual Skyrmions. This admixture will lead to additional attraction at intermediate distances. The purpose of this paper is to calculate 
the effect of this mixing on the N-N interaction for $N_{\mathrm{C}}=3$ starting with (10). The need for state mixing has been realized before [21] in the context of the product ansatz, but that ansatz is a poor starting point. Furthermore, as we state below, state mixing only makes sense for finite $N_{\mathrm{C}}$. At the same time there are algebraic finite $N_{\mathrm{C}}$ corrections to matrix elements that are difficult to include in the formalism of [21]. For these reasons, these early attempts to introduce state mixing did not find sufficient central attraction. We should remark here that a very different formalism, based on a study of fluctuations around the product ansatz 22] has been used to show that some attraction exists in two-pion range. Unfortunately the expansion underlying this approach fails in the region that is most crucial to central attraction; $1 \mathrm{fm}<R<2 \mathrm{fm}$.

For large $N_{\mathrm{C}}$, one can distinguish two energy scales or reciprocally two time scales. The slower time scale is associated with the motion in the collective manifold, i.e., $R$ and the orientation. The other time scale corresponds to the almost instantaneous response of the pion field to changes in $R$ and the relative isospin orientation. For large $N_{\mathrm{C}}$ we cannot separate the time scales for the two sets of adiabatic modes, as can be seen in the highly correlated doughnut. For $N_{\mathrm{C}}$ equal to three the situation changes. The $R$ motion is typically much slower than the rotational motion which leads to the separation of the nucleon and $\Delta$ states. We thus have three energy scales, that of the pion field, of the $N-\Delta$ separation and of the $R$ motion. We can now calculate a Born-Oppenheimer potential for the $R$-motion, which constitutes the slowest degree of freedom.

\section{A. Perturbative approach}

All of the effects of quick response of the pion field at each $R$ are already in Eq. (10). It is the effect of the rotational states we want to include. As a guide, we begin by studying it perturbatively. Let us call the full potential between two nucleons, including the effects of the rotational excited states, $V$. In terms of the Skyrmion interaction $v$ of (10) and to second order, $V$ is given by

$$
V(R)=\langle N N|v(R)| N N\rangle+\sum_{s}{ }^{\prime} \frac{\langle N N|v(R)| s\rangle\langle s|v(R)| N N\rangle}{E_{N N}(R)-E_{s}(R)} .
$$

(Here $E_{N N}$ is the two-nucleon energy and $E_{S}$ is the energy of the relevant excited state.) The first term on the right is the direct nucleon-nucleon projection of $v$ and is the term that has appeared in the literature. The second term is the correction due to rotational or excited states. It is clear from the energy denominator that the second term is attractive. For $N_{\mathrm{C}}=3$ the states $|s\rangle$ that can enter are $|N \Delta\rangle,|\Delta N\rangle$ and $|\Delta \Delta\rangle$. Except for a centrifugal term we shall add later, the excitation energy is assumed to be independent of $R$, and thus can be expressed in terms of the $\mathrm{N}-\Delta$ energy difference, $300 \mathrm{MeV}$. It is here that we are neglecting the effect of the position dependence of the inertial parameters.

The lowest order potential has been calculated by several groups using several levels of approximation to the Skyrme model [0, 9, 14]. The form found (or assumed), however, can always be cast in the form of the algebraic model given by [15]. This allows us to study both the large- $N_{\mathrm{C}}$ limit, as well as to include finite $N_{\mathrm{C}}$ effects explicitly in a systematic way. (The reader should note that the results in 90 include corrections for the tensor and spin-spin interactions.) 
The leading term in this expansion is given by the form (cf. (10))

$$
v(\vec{R})=v_{1}(r)+v_{2}(R) W+v_{3}(R) Z(\hat{R}) .
$$

The algebraic operators $W$ and $Z$ have very simple expectation value for nucleons, (see [15])

$$
\begin{aligned}
\langle N N|W| N N\rangle & =\frac{1}{9} P_{N}^{2}\left\langle N N\left|\sigma^{1} \cdot \sigma^{2} \tau^{1} \cdot \tau^{2}\right| N N\right\rangle, \\
\langle N N|Z| N N\rangle & =\frac{1}{9} P_{N}^{2}\left\langle N N\left|\left(3 \sigma^{1} \cdot \hat{R} \sigma^{2} \cdot \hat{R}-\sigma^{1} \cdot \sigma^{2}\right) \tau^{1} \cdot \tau^{2}\right| N N\right\rangle,
\end{aligned}
$$

i.e., apart from a finite $N_{\mathrm{C}}$ correction factor $P_{N}^{2}\left(P_{N}=1+2 / N_{\mathrm{C}}\right)$ they represent the spinspin and tensor interactions. If we use this form we find that the lowest order interaction in the nucleon-nucleon channel is given by

$$
V^{(0)}=v_{1}+\frac{v_{2} P_{N}^{2}}{9} \sigma^{1} \cdot \sigma^{2} \tau^{1} \cdot \tau^{2}+\frac{v_{3} P_{N}^{2}}{9}\left(3 \sigma^{1} \cdot \hat{R} \sigma^{2} \cdot \hat{R}-\sigma^{1} \cdot \sigma^{2}\right) \tau^{1} \cdot \tau^{2} .
$$

If we wish to evaluate the perturbation correction (11) we must evaluate the two sets of matrix elements $\langle N N|\tilde{v}(\vec{R})| N \Delta\rangle\langle N \Delta|\tilde{v}(\vec{R})| N N\rangle$ and $\langle N N|\tilde{v}(\vec{R})| \Delta \Delta\rangle\langle\Delta \Delta|\tilde{v}(\vec{R})| N N\rangle$ separately. Details are discussed in appendix A, but the final result for the first order correction to the $\mathrm{NN}$ interaction is

$$
\begin{aligned}
V_{\mathrm{PT}}^{(1)}= & -\frac{Q_{N}^{2}}{\delta}\left\{\left[\frac{1}{3} Q_{N}^{2} P_{0}^{\tau}+\left(\frac{16}{27} P_{N}^{2}+\frac{5}{27} Q_{N}^{2}\right) P_{1}^{\tau}\right]\left(v_{2}^{2}+2 v_{3}^{2}\right)+\right. \\
& \left(\sigma^{1} \cdot \sigma^{2}\right)\left[-\frac{1}{18} Q_{N}^{2} P_{0}^{\tau}+\left(\frac{16}{81} P_{N}^{2}-\frac{5}{162} Q_{N}^{2}\right) P_{1}^{\tau}\right]\left(v_{2}^{2}-v_{3}^{2}\right) \\
& \left.\left(3 \sigma^{1} \cdot \hat{R} \sigma^{2} \cdot \hat{R}-\sigma^{1} \cdot \sigma^{2}\right)\left[-\frac{1}{18} Q_{N}^{2} P_{0}^{\tau}+\left(\frac{16}{81} P_{N}^{2}-\frac{5}{162} Q_{N}^{2}\right) P_{1}^{\tau}\right]\left(v_{3}^{2}-v_{2} v_{3}\right)\right\} .
\end{aligned}
$$

Here $Q_{N}$ is an additional finite $N_{\mathrm{C}}$ correction factor

$$
Q_{N}=\sqrt{\left(1-1 / N_{\mathrm{C}}\right)\left(1+5 / N_{\mathrm{C}}\right)},
$$

$\delta$ is the N- $\Delta$ energy difference, and $P_{T}^{\tau}$ is a projection operator onto isospin $T$.

\section{B. Diagonalization of the interaction}

The perturbative expansion discussed in the previous subsection just gives a rough estimate of the effect of state mixing in the Born-Oppenheimer approximation. To solve for the Born-Oppenheimer Hamiltonian the total collective Hamiltonian is first divided into two parts, an intrinsic part and a part that only describes the radial motion,

$$
H=-\frac{\hbar^{2}}{2 M} R^{2} \partial_{R} R^{-2} \partial_{R}+\tilde{H}(R) .
$$

The hamiltonian $\tilde{H}(R)$ is nothing but the sum of the potential $v(R)$ we used in the perturbative calculation, plus the kinetic term used to calculate the energy difference in the energy denominators,

$$
K=\frac{1}{2 \Lambda}\left(I_{1}^{2}+I_{2}^{2}\right)+\frac{\hbar^{2}}{2 M R^{2}} L(L+1) .
$$


We now introduce the instantaneous eigenstates of the intrinsic Hamiltonian. For fixed $R$ we diagonalize $\tilde{H}$,

$$
\tilde{H}(R)|i\rangle_{R}=E_{i}(R)|i\rangle_{R}
$$

The standard Born-Oppenheimer approach is to start at large $R$, where the states are pure $N N$, and follow the continuous energy curve as $R$ becomes smaller to define the adiabatic potential energy. This is basically the procedure we follow, apart from a few additional complications encountered if we have more than one $N N$ state in any given channel. This is solved by using a model Hamiltonian to determine the unmixed adiabatic eigenstates, as will be discussed below.

Having defined the adiabatic eigenstates, the mathematically correct way to derive the adiabatic Hamiltonian is to decompose a general time-dependent solution of the Schrödinger equation in adiabatic eigenstates,

$$
|\Psi(t)\rangle=\sum_{i} a_{i}(R, t)|i\rangle_{R}
$$

If we substitute this in the time-dependent Schrödinger equation, and multiply from the left with ${ }_{R}\langle i|$, we find

$$
\begin{aligned}
i \dot{a}_{i} \delta_{i j}= & \delta_{i j}\left(\frac{-\hbar^{2}}{2 M} \frac{1}{R^{2}} \partial_{R} R^{2} \partial_{R}+E_{i}(R)\right) a_{i} \\
& +\frac{-\hbar^{2}}{M} \sum_{j}{ }_{R}\left\langle i\left|\partial_{R}\right| j\right\rangle_{R} \partial_{R} a_{j}(R, t) \\
& +\sum_{j}{ }_{R}\left\langle i\left|\frac{-\hbar^{2}}{2 M} \frac{1}{R^{2}} \partial_{R} R^{2} \partial_{R}\right| j\right\rangle_{R} a_{j}(R, t) .
\end{aligned}
$$

This expression exhibits both the adiabatic Hamiltonian (the first term on the right-hand side), and the corrections. If we assume there are no diagonal terms of $\partial_{R}$ between adiabatic eigenstates (since there are no closed circuits in $R$ space, it seems a reasonable assumption to ignore Berry's phase), the first correction describes a velocity dependent transition potential. The next term gives a non-adiabatic correction to the diagonal adiabatic potential, as well as a velocity independent transition potential.

\section{The potential in angular momentum coupled form}

To evaluate (11) it is convenient to introduce states $\left|I_{1} I_{2} L S J T\right\rangle$ labeled by the conserved (total) isospin $T$ and angular momentum $J$, as well as the NN channel orbital angular

momentum $L$ and spin $S$. Since we wish to evaluate the matrix elements of $\tilde{H}(R)$ in the basis $\left|I_{1}^{\prime} I_{2}^{\prime} L S J T\right\rangle$ we need to know the matrix elements of the potential $v$ in this basis. After performing some standard angular momentum algebra, we find that

$$
\begin{aligned}
& \left\langle I_{1} I_{2} L S J T|v| I_{1}^{\prime} I_{2}^{\prime} L S J T\right\rangle \\
& =v_{1} \delta_{S S^{\prime}} \delta_{L L^{\prime}} \delta_{I_{1} I_{1}^{\prime}} \delta_{I_{2} I_{2}^{\prime}}
\end{aligned}
$$




$$
\begin{aligned}
& +\frac{v_{2}}{9}(-1)^{S+T} \delta_{S S^{\prime}} \delta_{L L^{\prime}}\left\{\begin{array}{lll}
I_{1} & I_{2} & S \\
I_{2}^{\prime} & I_{1}^{\prime} & 1
\end{array}\right\}\left\{\begin{array}{ccc}
I_{1} & I_{2} & T \\
I_{2}^{\prime} & I_{1}^{\prime} & 1
\end{array}\right\}\left\langle I_{1}\left|\| T^{(11)}\right| I_{1}^{\prime}\right\rangle\left\langle I_{2}||\left|T^{(11)}\right| I_{2}^{\prime}\right\rangle \\
& +\frac{v_{3}}{9} \sqrt{30}(-1)^{L+L^{\prime}+S+J+T+I_{2}+I_{1}^{\prime}} \hat{L} \hat{L}^{\prime} \hat{S} \hat{S}^{\prime} \\
& \times\left(\begin{array}{lll}
L & 2 & L^{\prime} \\
0 & 0 & 0
\end{array}\right)\left\{\begin{array}{ccc}
S & L & J \\
L^{\prime} & S^{\prime} & 2
\end{array}\right\}\left\{\begin{array}{lll}
I_{1} & I_{2} & T \\
I_{2}^{\prime} & I_{1}^{\prime} & 1
\end{array}\right\}\left\{\begin{array}{ccc}
I_{1} & I_{2} & S \\
I_{1}^{\prime} & I_{2}^{\prime} & S^{\prime} \\
1 & 1 & 2
\end{array}\right\}\left\langle I_{1}||\left|T^{(11)}\right| I_{1}^{\prime}\right\rangle\left\langle I_{2}||\left|T^{(11)}\right| I_{2}^{\prime}\right\rangle .
\end{aligned}
$$

The relevant reduced matrix elements of $T$ are

$$
\begin{aligned}
\left\langle N||\left|T^{(11)}\right| N\right\rangle & =-10 \\
\left\langle\Delta||\left|T^{(11)}\right| \Delta\right\rangle & =-20 \\
\left\langle\Delta||\left|T^{(11)}\right| N\right\rangle & =-8 \sqrt{2} .
\end{aligned}
$$

The matrix elements of the kinetic part are taken to be very simple,

$$
\left\langle I_{1} I_{2} L S J T|K| I_{1}^{\prime} I_{2}^{\prime} L^{\prime} S^{\prime} J T\right\rangle=\delta_{I_{1} I_{1}^{\prime}} \delta_{I_{2} I_{2}^{\prime}} \delta_{L L^{\prime}} \delta_{S S^{\prime}}\left(300\left[I_{1}+I_{2}-1 / 2\right]+\frac{L(L+1)}{2 M_{I_{1} I_{2}} R^{2}}\right) .
$$

To account for the effects of centripetal repulsion at small $R$, we have added the centrifugal energy, $\hbar^{2} L(L+1) / 2 M_{I_{1} I_{2}} R^{2}$, to channel energies. We take $M_{I_{1} I_{2}}$ to be the reduced mass in the relevant channel,

$$
M_{I_{1} I_{2}}=\frac{M_{I_{1}} M_{I_{2}}}{M_{I_{1}}+M_{I_{2}}},
$$

where $M_{1 / 2}=932 \mathrm{MeV}$ and $M_{3 / 2}=1232 \mathrm{MeV}$.

For sake of comparison we shall also need the matrix elements of a model nucleon-nucleon interaction in the same channel (we have suppressed the spin and isospin projection quantum numbers),

$$
\begin{aligned}
\left\langle N N L S J T\left|V_{\mathrm{c}}^{T}+V_{\mathrm{s}}^{T} \sigma^{1} \cdot \sigma^{2}+V_{\mathrm{t}}^{T} \sigma_{i}^{1} \sigma_{j}^{2}\left(3 \hat{R}_{i} \hat{R}_{j}-\delta_{i j}\right)\right| N N L^{\prime} S^{\prime} J T\right\rangle \\
=\delta_{S S^{\prime}} \delta_{L L^{\prime}}\left(V_{\mathrm{c}}^{T}+V_{\mathrm{s}}^{T}(-1)^{1+S} 6\left\{\begin{array}{lll}
1 / 2 & 1 / 2 & S \\
1 / 2 & 1 / 2 & 1
\end{array}\right\}\right)+ \\
\quad 6 \sqrt{30} V_{\mathrm{t}}^{T}(-1)^{L+L^{\prime}+S+J} \hat{L} \hat{L}^{\prime} \hat{S} \hat{S}^{\prime}\left(\begin{array}{lll}
L & 2 & L^{\prime} \\
0 & 0 & 0
\end{array}\right)\left\{\begin{array}{ccc}
L & S & J \\
S^{\prime} & L^{\prime} & 2
\end{array}\right\}\left\{\begin{array}{ccc}
1 / 2 & 1 / 2 & S \\
1 / 2 & 1 / 2 & S^{\prime} \\
1 & 1 & 2
\end{array}\right\}
\end{aligned}
$$

Here we have chosen to use a parametrization identical to the one used in the discussion of perturbation theory.

In order to determine an effective potential from the analysis given above we perform a calculation that is standard in the Born-Oppenheimer approximation. We assume that at each $R$ we can diagonalize the potential. We then follow a state from large $R$, where it is purely made up out of nucleons, to small $R$ where we have mixing. This gives the usual adiabatic potential curves of molecular physics. A complication will be that we may have more than one nucleon state for given quantum numbers. We shall see that we can still fit the interaction in that case. 
The model space splits into two parts that are either symmetric or antisymmetric under interchange of the particles [23]. We select the antisymmetric part where the NN states satisfy the standard selection rule $L+S+T$ is odd. Of course, in contrast to the lowest order perturbative result, we will now find a channel dependent potential.

The fact that we have a different reduced mass in each channel will also lead to a position dependent effective mass. Suppose that $c_{I_{1} I_{2}}^{2}(R)$ gives the percentage of the $I_{1} I_{2}$ state in the diagonalization of the Born-Oppenheimer Hamiltonian. In that case the average radial kinetic energy is

$$
\sum_{I_{1} I 2}-\frac{\hbar^{2}}{2 M_{I_{1} I_{2}}} c_{I_{1} I_{2}}^{2}(R) \frac{1}{R^{2}} \partial_{R} R^{2} \partial_{R},
$$

and we can define a position dependent mass by

$$
M(R)=1 /\left(\sum_{I_{1} I 2} \frac{c_{I_{1} I_{2}}^{2}(R)}{M_{I_{1} I_{2}}}\right) .
$$

\section{Derivation of the potential}

Let us first consider the case $T=0$. A little analysis shows that in this case the two channels $J^{\pi}=1^{+}$and $J^{\pi}=1^{-}$give three two-nucleon states, enough to determine the three independent functions in (28). (There are no $N N$ states with $T=0$ and $J=0$.)

For $J^{\pi}=1^{+}$we have the two possible states $|N N L=0 S=1 J=1 T=0\rangle$ and $|N N L=2 S=1 J=1 T=0\rangle$. The matrix elements of (28) in this space take the form

$$
\left(\begin{array}{cc}
V_{\mathrm{c}}^{0}+V_{\mathrm{s}}^{0} & 2 \sqrt{2} V_{\mathrm{t}} \\
2 \sqrt{2} V_{\mathrm{t}} & V_{\mathrm{c}}^{0}+V_{\mathrm{s}}^{0}-2 V_{\mathrm{t}}^{0}
\end{array}\right),
$$

with eigenvalues

$$
E_{1}=V_{\mathrm{c}}^{0}+V_{\mathrm{s}}^{0}+2 V_{\mathrm{t}}^{0}, \quad E_{2}=V_{\mathrm{c}}^{0}+V_{\mathrm{s}}^{0}-4 V_{\mathrm{t}}^{0} .
$$

These numbers should be fitted to the two lowest eigenvalues of the diagonalization of the algebraic potential in the complete nucleon-delta space. If we also look at the state $|N N L=1 S=0 J=1 T=0\rangle$, with matrix element of (28) equal to

$$
E_{3}=V_{\mathrm{c}}^{0}-3 V_{\mathrm{s}}^{0}
$$

(again, this is equated to the lowest eigenvalue of the complete diagonalization) we find

$$
\begin{aligned}
& V_{\mathrm{c}}^{0}=E_{1} / 2+E_{2} / 4+E_{3} / 4, \\
& V_{\mathrm{s}}^{0}=E_{1} / 6+E_{2} / 12-E_{3} / 4, \\
& V_{\mathrm{t}}^{0}=\left(E_{3}-E_{2}\right) / 6 .
\end{aligned}
$$

A similar calculation for $T=1$ involves the three channels $J^{\pi}=0^{+}, 0^{-}, 1^{-}$(again $L+S+T=$ odd). The relevant matrix elements of (28) are 


$$
\begin{aligned}
E_{1}^{\prime} & \equiv\langle N N L=0 S=0 J=0 T=1|V| N N L=0 S=0 J=0 T=1\rangle \\
& =V_{\mathrm{c}}^{1}-3 V_{\mathrm{s}}^{1} \\
E_{2}^{\prime} & \equiv\langle N N L=1 S=1 J=1 T=1|V| N N L=1 S=1 J=1 T=1\rangle \\
& =V_{\mathrm{c}}^{1}+V_{\mathrm{s}}^{1}+2 V_{\mathrm{t}}^{1} \\
E_{3}^{\prime} & \equiv\langle N N L=1 S=1 J=0 T=1|V| N N L=1 S=1 J=0 T=1\rangle \\
& =V_{\mathrm{c}}^{1}+V_{\mathrm{s}}^{1}-4 V_{\mathrm{t}}^{1} .
\end{aligned}
$$

Thus

$$
\begin{aligned}
V_{\mathrm{c}}^{1} & =E_{1}^{\prime} / 4+E_{2}^{\prime} / 2+E_{3}^{\prime} / 4, \\
V_{\mathrm{s}}^{1} & =-E_{1}^{\prime} / 4+E_{2}^{\prime} / 6+E_{3}^{\prime} / 12, \\
V_{\mathrm{t}}^{1} & =\left(E_{2}^{\prime}-E_{3}^{\prime}\right) / 6 .
\end{aligned}
$$

We subtract the centrifugal force from our potentials, since in general this is not taken to be included in the potential itself. We had to include it in our kinetic term, since we make the Born-Oppenheimer separation between the radial motion and everything else, including the orbital motion.

\section{RESULTS}

As stated before, for each total isospin, $T=0,1$ we write $V$ in the $|N N L S J T\rangle$ space as

$$
\left\langle N N L S J T\left|V_{\mathrm{c}}^{T}+V_{\mathrm{s}}^{T} \sigma^{1} \cdot \sigma^{2}+V_{\mathrm{t}}^{T} \sigma_{i}^{1} \sigma_{j}^{2}\left(3 \hat{R}_{i} \hat{R}_{j}-\delta_{i j}\right)\right| N N L^{\prime} S J T\right\rangle .
$$

It is the $V_{\mathrm{c}}^{T}, V_{\mathrm{s}}^{T}$ and $V_{\mathrm{t}}^{T}$ that we study. We have extracted the necessary values of $v_{1}, v_{2}, v_{3}$ from the work of Walhout and Wambach [9]. This is an uncertain process for large $R$ since the potentials are quite small in this region and are calculated in [9] on a lattice by first calculating the total interaction energy of the $B=2$ system and then subtracting the rest energy of two free Skyrmions. We wish to compare the Skyrme based potential with a realistic nucleon-nucleon interaction. One cannot relate our result to modern effective potentials, such as the Bonn [24] and Paris [25] potentials, since their central parts contain explicit momentum dependent terms. For that reason we compare our potentials to the Reid soft core (RSC) potential [26].

In Figs. 1 and 2 we show the central potentials $V_{\mathrm{c}}^{T}$ calculated from Eq. (11), first from the first term on the right of (11) only (this is the result of Ref. [9]) and then with the Born-Oppenheimer method. Note we only show the result for intermediate $R, 1 \mathrm{fm}<$ $R<2 \mathrm{fm}$. To keep the figures simple we separate $T=0$ and $T=1$. The nucleons only result from [9] is independent of $T$ and shows a weak attraction. The perturbation result (15) gives considerably more attraction, which is in turn somewhat reduced by the full BornOppenheimer (BO) diagonalization. This reduction comes mostly from the centrifugal terms in the kinetic energy which were not included in the perturbative calculations, and partly from the higher order effects included in the diagonalization. The centrifugal terms reduce the mixing at small $R$, and hence lead to less central attraction. All the results, nucleons only, perturbation theory and $\mathrm{BO}$ diagonalization agree at large $R$. This reflects the fact that state mixing involves multiple pion exchange and hence has intermediate range. Also 
shown in Figs. 1 and 2 are the $T=0$ and $T=1$ components of the Reid soft core potential. They have less central attraction than we find. As we shall see that reflects a rather different shuffling of attraction between the central and the spin dependent interactions between the RSC and the Skyrme approach. But even at this level the fact that the combination of careful solution of the $B=2$ sector, state mixing and finite $N_{\mathrm{C}}$ effects gives too much attraction is a welcome change from the many previous attempts to derive the $N N$ interaction from the Skyrme approach which always found too little central attraction.

In Figs. 3 and 4 we show the $T=0$ and $T=1$ spin-dependent potentials. In these cases the nucleons-only potential, the perturbative results and the full diagonalization are all quite similar, but now the RSC is stronger than the Skyrme result, rather than weaker as it was in the central potential. This is the shuffling we referred to above. Figs. 5 and 6 show the tensor force for both values of isospin. Except for $T=1$ at small $R$ all the Skyrmion calculations and even the RSC agree. This reflects the dominance of one pion exchange for the tensor potential.

We see that though the effect of the Born-Oppenheimer corrections are modest for large $R$ they become large for intermediate $R$ in the central channel, but remain small in the other channels. The reason for this is that the corrections are small for all channels compared to the typical size of the potential, but that the lowest order central potential is much smaller than this typical size.

We saw in Figs. 1-4 that the RSC gives less central spin-independent attraction than we find but a stronger spin-dependent potential. It is therefore instructive to compare the more physical $S$-channel $L=0$ potentials. In Fig. 7 and 8 we show the ${ }^{3} S_{1}(T=0)$ and the ${ }^{1} S_{0}$ $(T=1)$ potentials. Now we see that the full Born-Oppenheimer potential looks quite similar to the RSC while the one obtained without mixing does not resemble the RSC. It is in this sense that we say that the present results give a credible account of the nucleon-nucleon interaction at intermediate range.

We now turn to a more detailed analysis of the Born-Oppenheimer approximation, the adiabatic approximation and non-adiabatic corrections. We analyze a representative case, the $J=1^{-}, T=1$ channel, since it mixes $N N, N \Delta$ and $\Delta \Delta$ states. The relevant states in the diagonalization are $|N N, L=1, S=1\rangle,|N \Delta, L=1, S=1\rangle,|N \Delta, L=1, S=2\rangle$, $|\Delta \Delta, L=1, S=1\rangle$ and $|\Delta \Delta, L=1, S=3\rangle$. In Fig. 9 we plot the energy of the adiabatic eigenstates, which remain distinct for all separations, except for a narrow avoided crossing near $1.2 \mathrm{fm}$. For this channel all the energies are repulsive. In Fig. 10 we show the percentage of each component in the lowest adiabatic eigenstate. It is this state that was used in the determination of the $N N$ potential. One can see that the $N N$ component is dominant, and we have only small admixtures of the other components. For small $R$ it is the third adiabatic eigenstate that admixes most strongly.

In Fig. 11 we show the effective mass of the lowest adiabatic eigenstate as defined by Eq. (30). Since the only effect on the masses we include is the centrifugal term, the mass increases with decreasing $R$. Preliminary calculations of the mass parameters for the classical $B=2$ system give mass parameters that decrease with decreasing $R$, at least in the attractive channel [20]. Thus the results of Fig. 11 should be taken only to remind us that the Skyrme approach leads to a picture of nucleons that distort and change their mass as they interact.

Corrections to the adiabatic approximation lead to transition potentials among the adiabats. The relevant matrix elements can be calculated exactly using standard algebraic 
techniques:

$$
{ }_{R}\left\langle i\left|\partial_{R}\right| j\right\rangle_{R}={ }_{R}\left\langle i\left|\left(\partial_{R} \tilde{H}(R)\right)\right| j\right\rangle_{R} /\left(E_{i}(R)-E_{j}(R)\right)
$$

We further assume that the (imaginary) diagonal matrix element is zero, which corresponds to a consistent real choice of $|j\rangle_{R}$. The second derivatives can now be evaluated using a sum over intermediate states. As argued in the previous section we have two terms, a local transition potential and a velocity dependent potential. We continue to study the $\mathrm{J}=1^{-}$, $T=1$ channel and consider the transition from the $N N$ adiabat to each of the five others. The local potentials for these transitions are shown in Fig. 12 as a function of $R$. They are on average much smaller than the difference between the relevant adiabatic eigenvalues, as they should be if the adiabatic approximation works. Near $R=1.2 \mathrm{fm}$ there is a spectacular excursion of the velocity dependent potential in Fig. 12. This can be traced back to the narrowly avoided level crossing in Fig. 9. This picture would suggest that at high $N N$ energy there should be the onset of strong $\Delta$ production. The theory is still too crude to make a detailed prediction, but this aspect of $N N$ dynamics suggested by the Skyrme approach deserves further investigation. The velocity dependent potential are a little more difficult to interpret. We have chosen to plot, in Fig. 13, the potential such that we have to multiply it by $-i$ times the scaled radial velocity $-i \beta=v / c=\frac{\hbar}{M c} \partial_{R}$. As can be seen this potential is very small for non-relativistic velocities.

\section{SUMMARY AND CONCLUSIONS}

Here we have shown that the Skyrme model can give strong mid-range nucleon-nucleon attraction that is in qualitative agreement with phenomenological potentials. This is in sharp contrast to early results based on the product ansatz. Two components play a key role in obtaining the attraction. First one must pay attention to the non-linear nature of the Skyrme Lagrangian, as shown in Refs. [9, 14, but second one must also include configuration mixing at intermediate distances as we have stressed here. This mixing is not easily formulated in the large $N_{\mathrm{C}}$ limit, but is easily included for $N_{\mathrm{C}}=3$, and hence brings with it some other finite $N_{\mathrm{C}}$ effects. The combined effect of the careful treatment of the non-linear equations and the configuration mixing is to give substantial central mid-range attraction for the NN system that is in qualitative agreement with the data. To go from this work to a theory that can be confronted with experiment in detail is a difficult challenge. There are $R$ dependent corrections to inertial parameters to include, there are dynamical quantum corrections, there are non-adiabatic effects that are particularly important at small $R$, and there are other mesons to include in the Skyrme Lagrangian. All these are under study. The success so far encourages us to proceed and to suggest that the results obtained so far will be robust under these refinements.

The success of the Born-Oppenheimer approach to the nucleon-nucleon interaction in the Skyrme model raises interesting questions about what "nucleons" are when they are interacting. We find that the state that is two nucleons at large separation becomes, adiabatically, a state with delta components as the nucleons approach. For the Skyrmion-Skyrmion system itself this distortion and deformation under interaction is even more dramatic. What then should we say about the interacting nucleons? That there is a big delta component in their 
wave function? That they are distorted by the interaction? In some sense this is true, but in the spirit of the Born-Oppenheimer approximation, it is not. The adiabatic potential derived from the Born-Oppenheimer prescription involves state mixing, in fact much of its attraction comes from that mixing, but that adiabatic potential is to be used as an effective potential between nucleons. Think of the van der Waals potential between hydrogen atoms. It comes from virtual excitation of the atoms, principally to the first $L=1$ state, but the potential is to be used between atoms in their ground state. One usually does not talk about the percent of atomic $L=1$ state in the hydrogen molecule. Following that example, we should not talk about the percent of delta in the state of two interacting nucleons. On the other hand, one could take apart the Born-Oppenheimer calculation and quote such a percent. It is really a matter of what basis one uses. Many discussion of delta's in the nucleus are unclear about this ambiguity. Our calculation helps to emphasize the perspective dependence of these discussions. We plan to return to this topic both for the nucleon-nucleon scattering system and for the deuteron in a subsequent paper, as well as to the question of the interaction effects on the nucleon mass parameter. We have also seen that corrections to the adiabatic approximation make it possible to compute transition potentials. These can be used to calculate Delta production in $N N$ collisions. In this paper we have only scratched the surface of this interesting problem.

In summary we have shown that including the effects of channel coupling ( $\Delta$ mixing) in the projection of the nucleon-nucleon interaction from recent studies of the potential in baryon number two Skyrmion systems, substantially increases the strength of the midrange central attraction bringing it into qualitative agreement with experiment. To obtain this result we introduce some quantum corrections (equivalently corrections for the finite number of colors). Many more sophisticated quantum corrections remain to be made. But our results show that a non-perturbative approach to the problem of obtaining the "static" nucleon-nucleon interaction from QCD based on the Skyrme approach has great promise. We are therefore encouraged to follow up the first success reported here.

\section{ACKNOWLEDGMENTS}

We wish to acknowledge Atsushi Hosaka for his contributions in the initial stages of this work. We also would like to thank Jochem Wambach for stimulating discussions.

This work was partially supported by the U.S. National Science Foundation.

\section{APPENDIX A: DETAILS OF THE PERTURBATIVE EXPANSION}

We exhibit details of the calculation for the first matrix element, for the part of $\tilde{H}$ proportional to $W$. We again make use of an equation from [15], Eq. (30).

$$
\begin{aligned}
& \langle N N|W| N \Delta\rangle\langle N \Delta|W| N N\rangle \\
& =\left\langle N\left|T_{p i}^{\alpha}\right| N\right\rangle\left\langle N\left|T_{q j}^{\alpha}\right| N\right\rangle\left\langle N\left|T_{p i}^{\beta}\right| \Delta\right\rangle\left\langle\Delta\left|T_{q j}^{\beta}\right| N\right\rangle \\
& =\left\langle N\left|T_{p i}^{\alpha}\right| N\right\rangle\left\langle N\left|T_{q j}^{\alpha}\right| N\right\rangle\left(\left\langle N\left|T_{p i}^{\beta} T_{q j}^{\beta}\right| N\right\rangle-\left\langle N\left|T_{p i}^{\beta}\right| N\right\rangle\left\langle N\left|T_{q j}^{\beta}\right| N\right\rangle\right) \\
& =\left\langle N N\left|\frac{N_{\mathrm{C}}^{4}}{9} \tau_{p}^{1} \sigma_{i}^{1} \tau_{q}^{1} \sigma_{j} P_{N}^{2}\left\{\frac{1}{2} Q_{N}^{2}\left(\frac{2}{3} \delta_{i j}-\frac{i}{3} \epsilon_{i j l} \sigma_{l}^{2}\right)\left(\frac{2}{3} \delta_{p q}-\frac{i}{3} \epsilon_{p q r} \tau_{r}^{2}\right)\right\}\right| N N\right\rangle
\end{aligned}
$$




$$
=\left\langle N N\left|N_{\mathrm{C}}^{4} \frac{P_{N}^{2} Q_{N}^{2}}{18}\left(2+\frac{2}{3} \sigma^{1} \cdot \sigma^{2}\right)\left(2+\frac{2}{3} \tau^{1} \cdot \tau^{2}\right)\right| N N\right\rangle
$$

Here we have used the finite- $N_{\mathrm{C}}$ correction terms $P_{N}$, defined before, and $Q_{N} \equiv$ $\sqrt{\left(1-1 / N_{\mathrm{C}}\right)\left(1+5 / N_{\mathrm{C}}\right)}$. In this manner we find that

$$
\begin{gathered}
\langle N N|\tilde{H}(\vec{R})| N \Delta\rangle\langle N \Delta|\tilde{H}(\vec{R})| N N\rangle \\
=\langle N N| P_{N}^{2} Q_{N}^{2}\left(2+\frac{2}{3} \tau^{1} \cdot \tau^{2}\right)\left\{\frac{1}{9}\left(v_{2}^{2}+2 v_{3}^{2}\right)+\frac{1}{27}\left(v_{2}^{2}-v_{3}^{2}\right) \sigma^{1} \cdot \sigma^{2}+\right. \\
\left.\frac{1}{27}\left(v_{3}^{2}-v_{2} v_{3}\right)\left(3 \sigma^{1} \cdot \hat{R} \sigma^{2} \cdot \hat{R}-\sigma^{1} \cdot \sigma^{2}\right)\right\}|N N\rangle
\end{gathered}
$$

and for an intermediate state with two $\Delta$ 's,

$$
\begin{aligned}
& \langle N N|\tilde{H}(\vec{R})| \Delta \Delta\rangle\langle\Delta \Delta|\tilde{H}(\vec{R})| N N\rangle \\
& =\langle N N| Q_{N}^{4}\left(\frac{4}{3}-\frac{2}{9} \tau^{1} \cdot \tau^{2}\right)\left\{\frac{1}{3}\left(v_{2}^{2}+2 v_{3}^{2}\right)-\frac{1}{18}\left(v_{2}^{2}-v_{3}^{2}\right) \sigma^{1} \cdot \sigma^{2}-\right. \\
& \left.\quad \frac{1}{18}\left(v_{3}^{2}-v_{2} v_{3}\right)\left(3 \sigma^{1} \cdot \hat{R} \sigma^{2} \cdot \hat{R}-\sigma^{1} \cdot \sigma^{2}\right)\right\}|N N\rangle .
\end{aligned}
$$

When we use the fact that the energy needed for excitation of a single $\Delta$, which we denote by $\delta$, is half that for excitation of two $\Delta$ 's, we find

$$
\begin{aligned}
V_{\text {exact }}^{(2)}= & -\frac{Q_{N}^{2}}{\delta}\left\{\left[\left(\frac{4}{9} P_{N}^{2}+\frac{2}{9} Q_{N}^{2}\right)+\left(\frac{4}{27} P_{N}^{2}-\frac{1}{27} Q_{N}^{2}\right) \tau^{1} \cdot \tau^{2}\right]\left(v_{2}^{2}+2 v_{3}^{2}\right)+\right. \\
& \left(\sigma^{1} \cdot \sigma^{2}\right)\left[\left(\frac{4}{27} P_{N}^{2}-\frac{1}{27} Q_{N}^{2}\right)+\left(\frac{4}{81} P_{N}^{2}+\frac{1}{162} Q_{N}^{2}\right) \tau^{1} \cdot \tau^{2}\right]\left(v_{2}^{2}-v_{3}^{2}\right) \\
& \left.\left(3 \sigma^{1} \cdot \hat{R} \sigma^{2} \cdot \hat{R}-\sigma^{1} \cdot \sigma^{2}\right)\left[\left(\frac{4}{27} P_{N}^{2}-\frac{1}{27} Q_{N}^{2}\right)+\left(\frac{4}{81} P^{2}+\frac{1}{162} Q_{N}^{2}\right) \tau^{1} \cdot \tau^{2}\right]\left(v_{3}^{2}-v_{2} v_{3}\right)\right\} .
\end{aligned}
$$

Of course this can be rewritten by introducing spin and isospin projection operators,

$$
\begin{aligned}
P_{0}^{\tau} & =\frac{1-\tau_{1} \cdot \tau_{2}}{4}, \\
P_{1}^{\tau} & =\frac{3+\tau_{1} \cdot \tau_{2}}{4}, \\
\tau^{1} \cdot \tau^{2} & =-3 P_{0}^{\tau}+P_{1}^{\tau} .
\end{aligned}
$$

We thus get the form (subscripts now denote isospin projections)

$$
\begin{aligned}
V_{\text {exact }}^{(2)}= & -\frac{Q_{N}^{2}}{\delta}\left\{\left[\frac{1}{3} Q_{N}^{2} P_{0}^{\tau}+\left(\frac{16}{27} P_{N}^{2}+\frac{5}{27} Q_{N}^{2}\right) P_{1}^{\tau}\right]\left(v_{2}^{2}+2 v_{3}^{2}\right)+\right. \\
& \left(\sigma^{1} \cdot \sigma^{2}\right)\left[-\frac{1}{18} Q_{N}^{2} P_{0}^{\tau}+\left(\frac{16}{81} P_{N}^{2}-\frac{5}{162} Q_{N}^{2}\right) P_{1}^{\tau}\right]\left(v_{2}^{2}-v_{3}^{2}\right) \\
& \left.\left(3 \sigma^{1} \cdot \hat{R} \sigma^{2} \cdot \hat{R}-\sigma^{1} \cdot \sigma^{2}\right)\left[-\frac{1}{18} Q_{N}^{2} P_{0}^{\tau}+\left(\frac{16}{81} P_{N}^{2}-\frac{5}{162} Q_{N}^{2}\right) P_{1}^{\tau}\right]\left(v_{3}^{2}-v_{2} v_{3}\right)\right\} .
\end{aligned}
$$




\section{REFERENCES}

[1] T. H. R. Skyrme, Nucl. Phys. 31 (1962) 556

[2] I. Zahed and G. E. Brown, Phys. Rep. 142 (1986) 1; K. F. Liu (ed.), Chiral solitons, (World Scientific, Singapore, 1987).

[3] N. R. Walet, R. D. Amado, and A. Hosaka, Phys. Rev. Lett. 68, 3849 (1992).

[4] A. D. Jackson, C. Weiss and A. Wirzba, Nucl. Phys. A529, 741 (1991).

[5] N. S. Manton, Phys. Rev. Lett. 60, 1916 (1988).

[6] A. Jackson, A. D. Jackson and V. Pasquier, Nucl. Phys. A432, 567 (1985).

[7] R. Vinh Mau, M. Lacombe, B. Loiseau, W. N. Nottingham and P. Lisboa, Phys. Lett. 150B, 259 (1985). M. Lacombe, B. Loiseau, R. Vinh Mau, and W. N. Cottingham, ibid. 161B, 31 (1985); ibid. 169B, 121 (1986); Phys. Rev. Lett. 57, 170 (1986).

[8] This has been argued by Jackson et al [6] using the product ansatz. It is hard to assess the significance of this statement since the product ansatz does not apply at these very small distances.

[9] T. S. Walhout and J. Wambach, Phys. Rev. Lett. 67, 314 (1991).

[10] Some workers used state mixing in the context of the product ansatz [21], and many, including WW, used some finite $N_{\mathrm{C}}$ effects.

[11] A. Klein, N. R. Walet, and G. Do Dang, Ann. Phys. (NY) 208, 90 (1991) and references therein.

[12] M. F. Atiyah and N. Hitchin, The geometric quantization of magnetic monopoles, (Princeton University Press, Princeton, 1988).

[13] M. F. Atiyah and N. S. Manton, Phys. Lett. 222B, 438 (1989); N. S. Manton, in: Geometry of Low-Dimensional Manifolds : Proceedings of the Durham Symposium, S. K. Donaldson and C. B. Thomas, eds. (Cambridge University Press, Cambridge, 1990).

[14] A. Hosaka, M. Oka and R. D. Amado, Nucl. Phys. A530, 507 (1991).

[15] M. Oka, R. Bijker, A. Bulgac, and R. D. Amado, Phys. Rev. C 36, 1727 (1987).

[16] V. B. Kopeliovic and B. E. Stern, JETP Lett. 45, 203 (1987); J. J. M. Verbaarschot, T. S. Walhout, J. Wambach, H. W. Wyld, Nucl. Phys. A468, 520 (1987); N. S. Manton, Phys. Lett. 192B, 177 (1988); J. J. M. Verbaarschot, Phys. Lett. 195B, 235 (1987).

[17] It is not immediately clear whether one can describe the symmetries in the doughnut in this way. We have studied this problem in some detail and have been able to prove that this is possible, but we have not yet been able to derive a sensible physical mechanism for the partial symmetry restoration of the doughnut.

[18] R. D. Amado, R. Bijker and M. Oka, Phys. Rev. Lett. 58, 654 (1986).

[19] Note the close analogy to an axially deformed nucleus, which can be represented by a point on the sphere $S_{2}$. In both cases this represents a state that is not the most general deformed body for the group under consideration, which leads to selection rules, $K_{z}=0$ for the nuclear case, and $\vec{K}=\vec{I}+\vec{S}=0$ for the case of the Skyrmion.

[20] J. Wambach, private communication; A. Hosaka, unpublished result (contributed paper to "Baryons '92").

[21] A. Depace, H. Müther and A. Faessler, Phys. Lett. 188B, 307 (1987); G. Kälbermann and J. M. Eisenberg, J. Phys. G15, 157 (1989).

[22] H. Yamagishi and I. Zahed, Phys. Rev. D 45, 965 (1991); V. Thorsson and I. Zahed, Phys. Rev. D 45965 (1992); T. Otofuji, Y. Kondo, S. Saito and R. Seki, Phys. Rev. D, in press. 
[23] This corresponds to assuming either symmetry or antisymmetry between the individual Skyrmions. In the semiclassical quantization of the doughnut a similar problem is encountered, in terms of the Finkelstein-Rubinstein constraints (E. Braatten and L. Carson, Phys. Rev. D 38, 3525 (1988)).

[24] C. Holinde, R. Machleidt and C. Elster, Phys. Rep. 149, 1 (1987).

[25] M. Lacombe, B. Loiseau, J. M. Richard, R. Vinh Mau, J. Cote, D. Pires, and R. DeTourreil, Phys. Rev. C 21, 861 6q(1980).

[26] R. V. Reid jr., Ann. Phys. (NY) 50, 411 (1968). 


\section{FIGURES}

FIG. 1. The central potential $V_{\mathrm{c}}^{T}$ as a function of $R$ in the region 1 to $2 \mathrm{fm}$ for the $T=0$ channel. The solid line gives the nucleons only result of [9]. The longer dashed line is the result of the state mixing in perturbation theory and the shorter dashed line of the full Born-Oppenheimer diagonalization. The dotted line is the Reid soft core potential in this channel.

FIG. 2. Same as Figure 1 but for $T=1$.

FIG. 3. The spin dependent potential $V_{\mathrm{s}}^{T}$ as a function of $R$ in the region 1 to $2 \mathrm{fm}$ for the $T=0$ channel. The solid line gives the nucleons only result of [9]. The longer dashed line is the result of the state mixing in perturbation theory and the shorter dashed line of the full Born-Oppenheimer diagonalization. The dotted line is the Reid soft core potential in this channel.

FIG. 4. The same as Figure 3 but for the $T=1$ spin-dependent potential.

FIG. 5. The tensor potential $V_{\mathrm{t}}^{T}$ as a function of $R$ in the region 1 to $2 \mathrm{fm}$ for the $T=0$ channel. The solid line gives the nucleons only result of [9]. The longer dashed line is the result of the state mixing in perturbation theory and the shorter dashed line of the full Born-Oppenheimer diagonalization. The dotted line is the Reid soft core potential in this channel.

FIG. 6. The same as Figure 5 but for the $T=1$ tensor potential.

FIG. 7. The potential in the ${ }^{3} S_{1}(T=0)$ channel as a function of $R$. The solid line gives the nucleons only result of [9]. The dashed line is the result of state mixing in a full Born-Oppenheimer diagonalization, and the dotted line is the Reid soft core potential in this channel.

FIG. 8. Same as Figure 7 but for the ${ }^{1} S_{0}(T=1)$ channel.

FIG. 9. The Born-Oppenheimer energy eigenvalues coming from diagonalization of the adiabatic energy in the $J=1^{-}, T=1$ channel as a function of the adiabatic variable $R$. The lowest energy state goes over to the $N N$ state for large $R$. The next three states go over into the $N \Delta$ states, and the upper two into the $\Delta \Delta$ states.

FIG. 10. The percentage admixture of configurations in the lowest $(N-N)$ adiabatic state of the $J=1^{-} T=1$ channel as a function of $R$. The solid line is the $N N$ component, and the seecond largest component, given by the short-dashed line is the state $|N \Delta, L=1, S=2\rangle$.

FIG. 11. The effective mass of the "nucleon" in the lowest $J=1^{-} T=1$ channel as a function of $R$. This is taken to be twice the reduced mass. 
FIG. 12. The velocity independent transition potential from the lowest $(N N)$ adiabatic state of the $J=1^{-} T=1$ channel as a function of $R$ for transitions to each of the five other channels. The numbers in the legend refer to the order of the states in Fig. 9, where one is the lowest energy adiabatic state.

FIG. 13. Same as Figure 12 but for the velocity dependent transition potential. Each term is understood to be multiplied by $-i \beta$ where $\beta=v / c$. 